\title{
Transverse Mode Instability Analysis in Fibre Amplifiers
}

\author{
Michalis N. Zervas ${ }^{\mathrm{a}, \mathrm{b}}$
}

${ }^{a}$ Optoelectronics Research Centre, University of Southampton, Southampton SO17 1BJ, UK

${ }^{\mathrm{b}}$ Advanced Laser Laboratory, SPI Lasers UK Ltd, Southampton SO30 2QU, UK;

\begin{abstract}
Mode instabilities in fiber amplifiers are analyzed by a new approach, considering the stability of the steady state FM amplification in the presence of transverse amplitude and/or phase perturbations, taking into account the effects of population inversion and thermal loading due to quantum-defect heating. Population inversion contribution to instability is shown to be dominant at low powers and high inversion. Under high powers and low inversion (high amplifier saturation) the thermal effects dominate the instability behavior. A simple and easy to interpret TMI power threshold formula is derived for the first time.
\end{abstract}

Keywords: transverse mode instabilities, fiber amplifiers, fiber lasers, high power lasers, high power amplifiers.

\section{INTRODUCTION}

Power scaling in single-mode fiber lasers and amplifiers has been hindered by transverse modal instability (TMI) [1],[2]. TMI amounts to a threshold-like onset of transverse spatial mode competition that degrades the output beam quality [2]. TMI has been observed experimentally in a large variety of $\mathrm{Yb}^{3+}$-doped optical fibers under different pumping and seeding conditions (see [1] and references therein). In the case of broad linewidth operation ( $\Delta v>\sim 25 \mathrm{GHz}$ ), when plotted against the active fiber core diameter, the high power fiber amplifier TMI threshold appears to be largely inversely proportional to the core area (i.e. $\propto D^{-2}$ ), regardless of the type of fiber used (see Fig. 1; top data set and blue dashed line). However, in the case of narrow linewidth operation $(\Delta v<\sim 25 \mathrm{GHz})$, the dependence of the TMI threshold on the core diameters appears to be much more severe (see Fig. 1; lower data set - red dashed line), demonstrating that the seed linewidth has a strong effect on the TMI threshold [3]-[6].

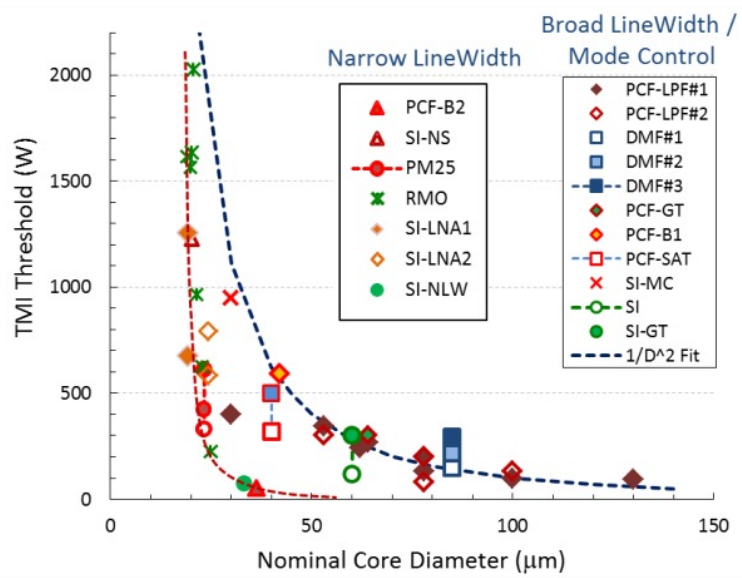

Fig. 1: High power fiber amplifier TMI threshold as a function of core diameter in the case of broad linewidth $(\Delta v>\sim 25 \mathrm{GHz}-$ top data set - blue dashed line), and narrow linewidth $(\Delta v<\sim 25 \mathrm{GHz}$ - lower data set - red dashed line).

In high power operation, the origin of this effect is considered to be predominantly the core thermally-induced refractive index changes and a number of theoretical models have been introduced to investigate the TMI characteristics. In this case, the combined core thermal load due to pump/signal quantum defect and photodarkening is considered to be the TMI driving force. However, there are big discrepancies in the quoted thermal load values at the TMI threshold, varying from $34 \mathrm{~W} / \mathrm{m}$ [7], down to $15 \mathrm{~W} / \mathrm{m}$ [8] and even $0.3-3 \mathrm{~W} / \mathrm{m}$ [9],[10] in the case of narrow linewidth operation. This clearly indicates that there are other contributions to the observed effects. In another study, it has been argued that under narrow-

Fiber Lasers XIV: Technology and Systems, edited by Craig A. Robin, Ingmar Hartl, Proc. of SPIE Vol. 10083, 100830M · @ 2017 SPIE · CCC code: 0277-786X/17/\$18 · doi: 10.1117/12.2252435 
linewidth and lower power operation, the inversion related refractive index changes are responsible for the observed TMI effects [9].

All theoretical models so far consider the interaction of two propagating modes and study the power exchange between these modes and TMI threshold either through Stimulated Thermal Rayleigh Scattering (STRS) static or dynamic mode interactions [11]-[19]. In this work, we consider the propagation of a single mode, i.e. the fundamental mode, and study the stability of the mode in the presence of a small perturbation. This approach provides a simple physical picture for the TMI process and results in a TMI threshold condition.

\section{MODEL OUTLINE}

\subsection{Paraxial Wave Approximation}

We consider the electric field of the fundamental mode (FM) $E=E_{s}(r, \varphi, z ; t) \exp \left[i\left(\beta_{s} z-\omega_{0} t\right)\right]$. The paraxial wave equation under the slowly-varying envelope approximation, accounting for the effects of both the inversion and thermal load on the fiber refractive index, takes the form:

$$
\frac{\partial E_{s}}{\partial z}+\frac{1}{v_{g}} \frac{\partial E_{s}}{\partial t}-\frac{i}{2 \beta_{s}}\left\{\nabla_{T}^{2}+\left[n_{0}^{2} k_{0}^{2}-\beta_{s}^{2}\right]\right\} E_{s}=\frac{i}{2 \beta_{s}} \omega_{0}^{2} \mu_{0} P_{N L}
$$

where $P_{N L}=\varepsilon_{0} \Delta \varepsilon_{N L} E_{s}$ is the nonlinear material polarization and $\Delta \varepsilon_{N L} \approx 2 n_{0} \Delta n_{N L} \cdot \nabla_{T}^{2}$ is the transverse Laplacian operator. The nonlinear refractive index $\Delta n_{N L}$ is given by $\Delta n_{N L}=\Delta n_{T}+\Delta n_{N}$ with the thermally- and inversion-induced refractive-index changes given by:

$$
\begin{aligned}
& \Delta n_{T}(r, \varphi, z ; t)=\left(\frac{d n}{d T}\right) \Delta T(r, \varphi, z ; t) \\
& \Delta n_{N}(r, \varphi, z ; t)=-\frac{i}{2 k_{0}}\left[\left(\sigma_{e s}+\sigma_{a s}\right)(1+i a) N_{2}(r, \varphi, z ; t)-\sigma_{a s} N_{0}(r, \varphi, z ; t)\right]
\end{aligned}
$$

respectively, where $\Delta T$ is the core temperature change, $(d n / d T)$ is the thermo-optic coefficient, $N_{2}$ is the excited-state population of $\mathrm{Yb}^{3+}, N_{0}$ is the total $\mathrm{Yb}^{3+}$ concentration, $\sigma_{\mathrm{es}}\left(\sigma_{\mathrm{as}}\right)$ is the signal emission (absorption) cross-section and $a$ is the ratio of the real and imaginary parts of susceptibility [20].

The variations of excited state population $N_{2}$ are given by:

$$
\frac{d N_{2}}{d t}=-\frac{N_{2}}{\tau}-\left[\left(\sigma_{a p}+\sigma_{e p}\right) N_{2}-\sigma_{a p} N_{0}\right] \Phi_{p}-\left[\left(\sigma_{a s}+\sigma_{e s}\right) N_{2}-\sigma_{a s} N_{0}\right] \Phi_{s}
$$

where $\Phi_{p}=I_{p} /\left(h v_{p}\right)$ and $\Phi_{s}=I_{s} /\left(h v_{s}\right)$ are the total pump and signal photon fluencies, and $\tau$ is the excited state lifetime, and $I_{\mathrm{p}(\mathrm{s})}$ is the pump (signal) intensity. The temperature change $\Delta T$ is given by the heat equation:

$$
\rho_{0} C_{0} \frac{d \Delta T}{d t}-\kappa_{0} \nabla_{T}^{2}(\Delta T)=Q_{T}
$$

where $\rho_{0}, C_{0}, \kappa_{0}$ are the density, the specific heat capacity, and the thermal conductivity of the fiber material, respectively, and $Q_{\mathrm{T}}$ is the heat power density given by: 


$$
Q_{T}=q_{D} \frac{d I_{s}}{d z}+\alpha_{s} I_{s}
$$

where $q_{D}=\left(\lambda_{s} / \lambda_{p}-1\right)$ is the quantum defect and $\alpha_{\mathrm{s}}$ the signal background loss.

\subsection{Linear Stability Analysis}

A TMI condition is derived by first linearizing Equations (1), (4) and (5). The electric field, inversion and thermal distributions are perturbed from their steady-state values:

$$
\begin{aligned}
& E_{s}=E_{s s}+\varepsilon(r, \varphi, z ; t) \\
& N_{2}=N_{2}^{s s}+\delta n_{2}(r, \varphi, z ; t) \\
& \Delta T=\Delta T_{s s}+\delta T(r, \varphi, z ; t)
\end{aligned}
$$

where $E_{s s}, N_{2}^{s s}, \Delta T^{s s}$ are the steady-state values for the electric field, excited-state population and core temperature, respectively, while $\varepsilon, \delta n_{2}, \delta T$ are the corresponding transverse perturbations. It is assumed that $\varepsilon<<E_{s s}, \delta n_{2}<<N_{2}^{s s}, \delta T<<\Delta T^{s s}$. The stability of the transverse perturbations is studied, using standard perturbation techniques. For this, we use the ansatz that transverse perturbations in cylindrical coordinates are proportional to

$$
\begin{aligned}
& \varepsilon(r, \varphi, z ; t)=\left(\varepsilon_{1}+i \varepsilon_{2}\right) J_{m}\left(u_{\varepsilon} r\right) e^{i m \varphi} e^{i\left(K_{z} z-\Omega t\right)} \\
& \delta n_{2}(r, \varphi, z ; t)=\delta n_{20} J_{m}\left(u_{\varepsilon} r\right) e^{i m \varphi} e^{i\left(K_{z} z-\Omega t\right)} \\
& \delta T(r, \varphi, z ; t)=\delta T_{0} J_{m}\left(u_{\varepsilon} r\right) e^{i m \varphi} e^{i\left(K_{z} z-\Omega t\right)}
\end{aligned}
$$

where $J_{\mathrm{m}}\left(u_{\varepsilon} r\right) \exp (\mathrm{i} m \varphi)$ describes the transverse dependence, $K_{\mathrm{Z}}$ is the longitudinal propagation constant and $\Omega$ is the frequency of the perturbation.

By substituting Eqn. (8) into the linearized equations, we obtain an algebraic system of two equations for $\varepsilon_{1}$ and $\varepsilon_{2}$, for which the requirement for non-trivial solutions results in the following dispersion relation:

$$
X^{2}-i P_{10} X-Y\left(Y-P_{20}\right)=0
$$

where:

$$
\begin{aligned}
& X=K_{z}-\frac{\Omega}{v_{g}} \\
& Y=\frac{u_{T}^{2}}{2 \beta_{s}} ; \quad u_{T}^{2}=u_{\varepsilon}^{2}-u_{s}^{2} \\
& P_{10}=G+2 k_{0}\left(\frac{\partial n}{\partial T}\right) N I_{s} ; \quad P_{20}=-G a_{e f f}+2 k_{0}\left(\frac{\partial n}{\partial T}\right) M I_{s}
\end{aligned}
$$

The other parameters are given by:

$$
g_{s}=\left(\sigma_{a s}+\sigma_{e s}\right) N_{2}^{s s}-\sigma_{a} N_{0} ;
$$




$$
\begin{gathered}
G=g_{s}\left(\frac{I_{s}}{I_{s}^{s a t}}\right) \frac{A-a \Omega \tau}{A^{2}+(\Omega \tau)^{2}} ; \quad a_{e f f}=\frac{a A+\Omega \tau}{A-a \Omega \tau} \\
A=1+\frac{I_{p}}{I_{p}^{s a t}}+\frac{I_{s}}{I_{s}^{\text {sat }}} ; \quad B=\left(\frac{g_{s}}{\sigma_{a s}+\sigma_{e s}}\right) \\
C=\frac{A B\left(1 / I_{s}^{s a t}\right)}{A^{2}+(\Omega \tau)^{2}} ; \quad D=\frac{\Omega \tau B\left(1 / I_{s}^{s a t}\right)}{A^{2}+(\Omega \tau)^{2}} \\
E=q_{D}\left(\sigma_{a s}+\sigma_{e s}\right) I_{s} ; \quad F=\left(q_{D} g_{s}+\alpha_{s}\right) \\
M=\frac{(F-E C) \kappa u_{\varepsilon}^{2}+D E\left(\Omega \rho_{0} C_{0}\right)}{\left(\kappa u_{\varepsilon}^{2}\right)^{2}+\left(\Omega \rho_{0} C_{0}\right)^{2}} ; \quad N=\frac{(F-E C)\left(\Omega \rho_{0} C_{0}\right)-D E \kappa u_{\varepsilon}^{2}}{\left(\kappa u_{\varepsilon}^{2}\right)^{2}+\left(\Omega \rho_{0} C_{0}\right)^{2}}
\end{gathered}
$$

$I_{s}^{s a t}=h v_{s} /\left[\tau\left(\sigma_{a s}+\sigma_{e s}\right)\right]$ and $I_{p}^{s a t}=h v_{p} /\left[\tau\left(\sigma_{a p}+\sigma_{e p}\right)\right]$ are the signal and pump saturation intensities.

From Eqn. (9) we obtain for the effective propagation constant of the perturbation:

$$
X_{ \pm}=\left(+i P_{10} \pm \sqrt{4 Y\left(Y-P_{20}\right)-P_{10}^{2}}\right) / 2
$$

The parameters $Y, P_{10}$, and $P_{20}$ contain contributions from both the inversion and the thermal loading. The perturbation power gain is given by the imaginary part of $X_{ \pm}$, namely, $g_{\varepsilon}=-2 \operatorname{Im}\left(K_{z}\right)$. The instability gain coefficient is then given by:

$$
g_{T M I}=\operatorname{Re}\left[\sqrt{P_{10}^{2}-4 Y\left(Y-P_{20}\right)}\right]-P_{10}
$$

Eqn. (20) provides the local TMI gain coefficient as a function of the local steady-state signal intensity $I_{s}$ and excitedstate population $N_{2}^{s s}$. In an amplifier, however, these parameters and, therefore, the TMI gain coefficient vary along the amplifier length. The total instability gain is then obtained by integrating $g_{\text {TMI }}$ along the entire amplifier length $L$, namely:

$$
G_{T M I}=\int_{0}^{L} g_{T M I}(z) d z
$$

\section{RESULTS}

\subsection{TMI gain coefficient under constant power, inversion and temperature}

Significant physical insight and understanding of the interplay between signal and pump intensities, inversion and thermal load and their contribution of TMI gain can be obtained by considering these parameters as constants. Fig. 1(a) plots the instability gain coefficient as a function of the instability frequency $(\Omega)$ for fibre core/cladding diameters of $10 \mu \mathrm{m} / 125 \mu \mathrm{m}$. The signal power $\left(P_{\mathrm{s}}\right)$ is $0.1 \mathrm{~W}$ and the pump power $\left(P_{\mathrm{p}}\right)$ varies from $0.125 \mathrm{~W}$ to $1 \mathrm{~W}$. It is shown that the instability gain coefficient increases with pump power. Fig. 1(b) plots the instability gain coefficient as function of the instability frequency $(\Omega)$ for $P_{\mathrm{s}}=0.1 \mathrm{~W}$ and $P_{\mathrm{p}}=1 \mathrm{~W}$ when inversion only, thermal only and combined inversion \& thermal effects 
are considered. In the case of small core diameter and low signal regime, the instability is dominated by inversion related refractive index changes, and the instability gain peak is obtained for $\Omega>0$, in close agreement with Ref. [9].

0
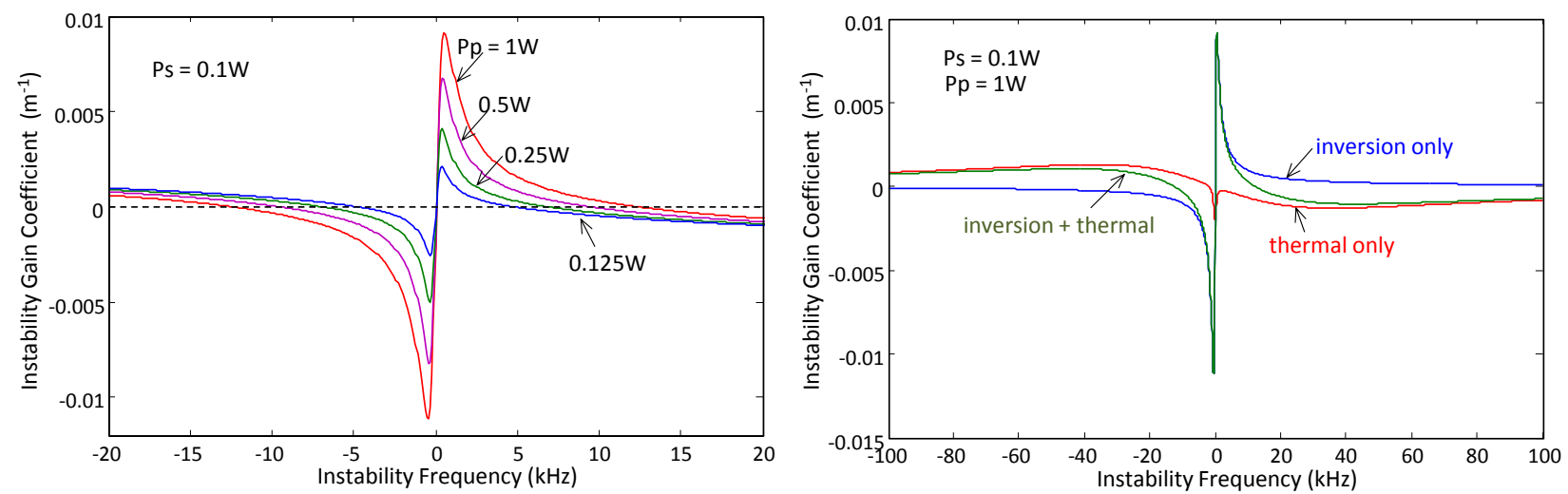

Fig. 1: Instability gain coefficient versus instability frequency $(\Omega)$ for (a) different pump powers and $P_{\mathrm{s}}=0.1 \mathrm{~W}$, (b) for $P_{\mathrm{s}}=0.1 \mathrm{~W}$ and $P_{\mathrm{p}}=1 \mathrm{~W}$ when inversion only, thermal only and combined inversion $\&$ thermal effects are considered $(\mathrm{Dc} / \mathrm{Dcl}=10 \mu \mathrm{m} / 125 \mu \mathrm{m})$.

A different response develops when the signal/pump power and core diameter are increased. Fig. 2(a) plots the instability gain coefficient for $\mathrm{Dc} / \mathrm{Dcl}=50 \mu \mathrm{m} / 500 \mu \mathrm{m}$ and $P_{\mathrm{p}}=1 \mathrm{~kW}$ for different signal powers. It is shown that in high power operation instability gain peak appears for $\Omega<0$. Fig. 2(b) plots the instability gain as a function of the instability frequency, for $P_{\mathrm{p}}=1000 \mathrm{~W}$ and $P_{\mathrm{s}}=10 \mathrm{~W}$ when inversion only, thermal only and combined inversion \& thermal effects are considered. It is shown that in this regime of operation TMI is predominantly due to thermal effects. In contrast with the low power regime, in this case TMI gain is observed for $\Omega<0$ (Stokes shift) and frequency shifts of a few kHz, in agreement with previous analyses [12].
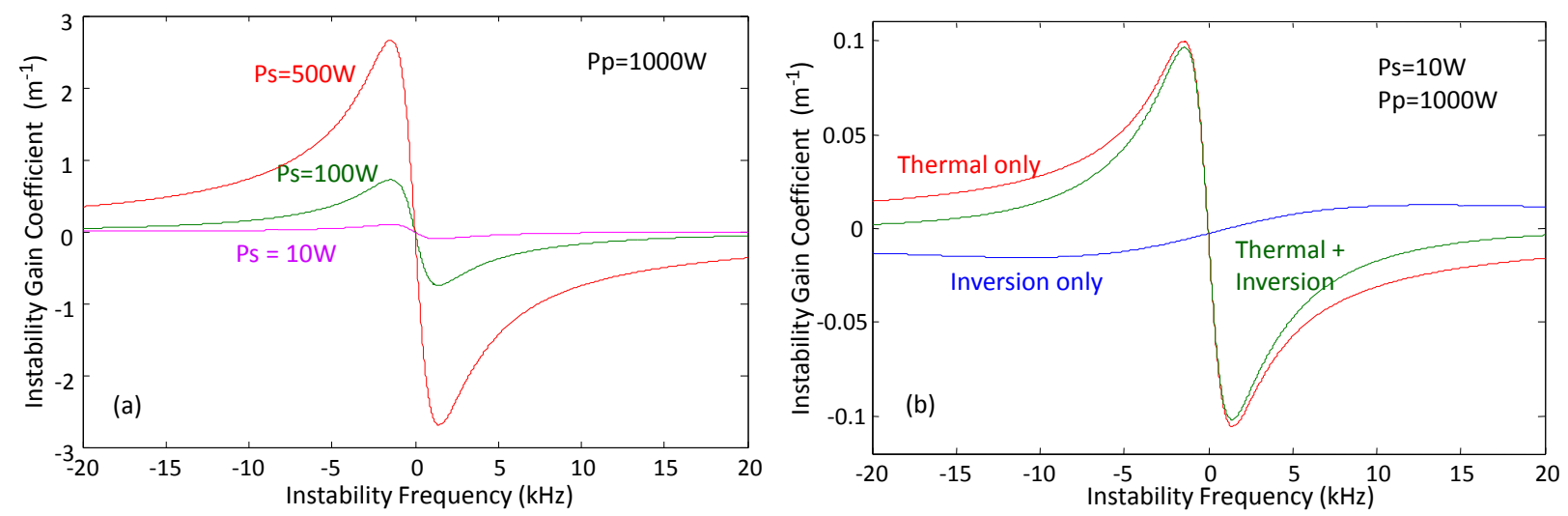

Fig. 2: Instability gain coefficient versus instability frequency $(\Omega)$ for (a) $\mathrm{Pp}=1000 \mathrm{~W}$ and different signal powers; (b) for $P_{\mathrm{s}}=$ $10 \mathrm{~W}$ and $P_{\mathrm{p}}=1 \mathrm{~kW}$ when inversion only, thermal only and combined inversion $\&$ thermal effects are considered

$(\mathrm{Dc} / \mathrm{Dcl}=50 \mu \mathrm{m} / 500 \mu \mathrm{m})$.

\subsection{TMI Gain Distribution - Comparison with Literature}

We next compare our TMI gain calculations with other STRS gain calculations and published data [12]. Fig. 3(a) and (b) show the pump power and signal distribution along the fiber amplifier length, while Fig. 3(c) and (d) show the local gain and heat load distributions, respectively. Fig. 4(a) and (b) show the instability gain coefficient (Eqn. (20)) as a function of amplifier length and instability frequency offset in the case of thermal and inversion only contributions, respectively. It is shown that the thermal-only contribution to the TMI gain peaks close to the amplifier input, where the heat load is maximum, for Stokes shifts $(\Omega<0)$ of a few $\mathrm{kHz}$. The inversion-only contribution peaks again close to the amplifier input, 
where the inversion is maximum, but for anti-Stokes shifts $(\Omega>0)$ of a few 100's kHz. Fig. 4(c) shows the combined thermal and inversion related contributions to the TMI gain coefficient distribution. It should be mentioned that the gain coefficient in all cases is highly localized.
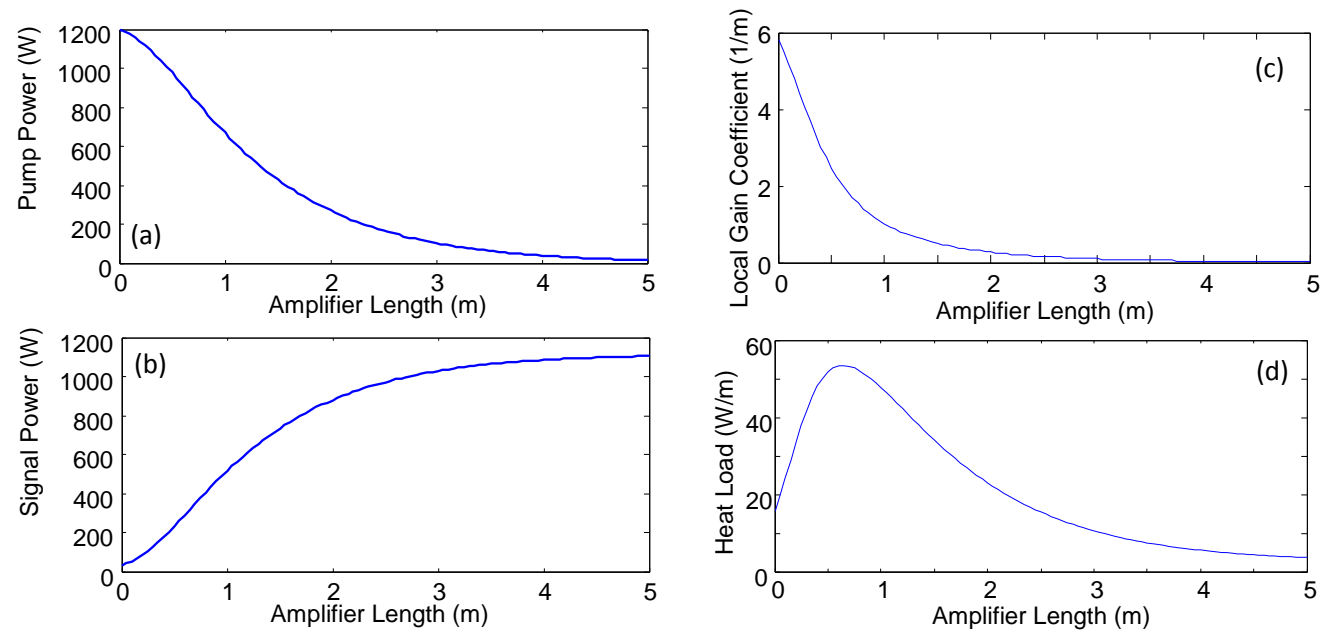

Fig. 3: (a) pump power distribution (b) signal power, (c) local gain coefficient and (d) heat load as a function of amplifier length (data from [12])

(a)

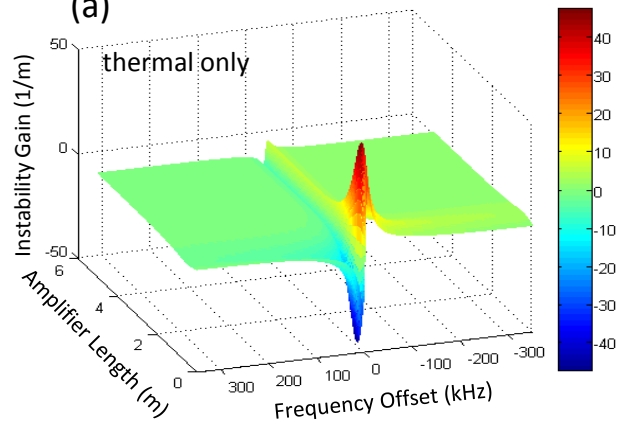

(b)

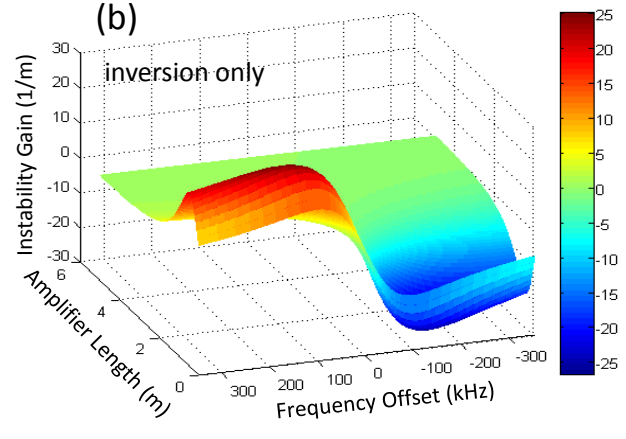

(c)
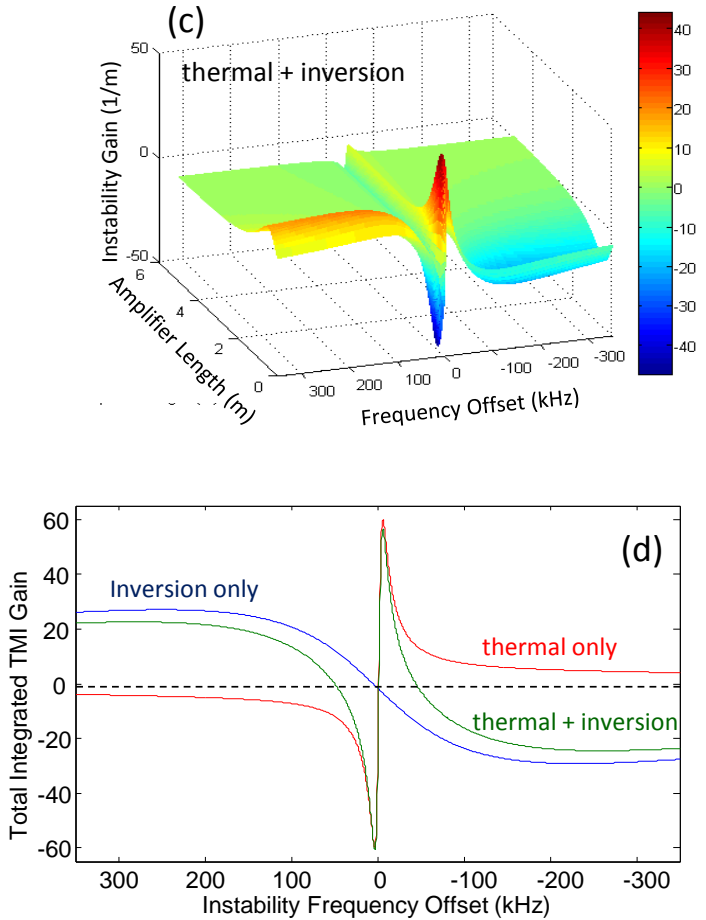

Fig. 4: (a) pump power distribution (b) signal power, (c) local gain coefficient and (d) heat load as a function of amplifier length (data from [12])

Fig. 4(d) shows the corresponding TMI gain, given by Eqn. (21). It is shown that the total gain/loss spectrum (thermal + inversion) is reduced due to the fact that the individual contributions have opposite signs. However, the TMI gain peak is dominated by the thermal effects, due to the fact that the inversion-only contributions are very small for small frequency offsets. The peak gain is $\sim 60$ for $\Omega \approx-5 \mathrm{kHz}$, in close agreement with [12]. 


\section{TMI POWER THRESHOLD}

The instability threshold is obtained from Eqn. (19) when the following instability condition is fulfilled:

$$
4 Y\left(Y-P_{20}\right)-P_{10}^{2} \leq 0
$$

where $Y, P_{10}$ and $P_{20}$ are given by Eqn.s (11) and (12). In the case of high power and high saturation (low inversion) operation, where thermal effects dominate, the instability condition (22) leads to a simplified TMI Power Threshold [21]:

$$
P_{T M I}^{t h r}=\frac{\kappa_{0} U_{\varepsilon}^{2}\left(U_{\varepsilon}^{2}-U_{s}^{2}\right)}{4 \pi n_{\text {eff }}\left(\frac{\partial n}{\partial T}\right) \alpha_{s}^{\prime}}\left(\frac{\lambda_{s}}{D_{0}}\right)^{2}
$$

Where in the high power regime $\alpha_{s}^{\prime}$ is approximated as $\alpha_{s}^{\prime} \cong \alpha_{s}+0.5 q_{D} g_{s} . U_{\varepsilon}$ and $U_{\mathrm{s}}$ are the transverse wavenumbers of the perturbation and fundamental mode (FM), respectively, $\kappa_{0}$ is the glass thermal conductivity, $n_{\text {eff }}$ is the FM effective index, $q_{\mathrm{D}}$ is the quantum defect, $g_{\mathrm{s}}$ is the FM saturated gain coefficient, while $\alpha_{\mathrm{s}}$ is the background loss coefficient (including photodarkening). $\lambda_{\mathrm{s}}$ is the signal wavelength and $D_{0}$ is the core diameter. For $P \geq P_{\text {th }}$ small transverse amplitude and/or phase perturbations grow exponentially, scatter light into to HOMs and lead to TMI. For $P<P_{\text {th }}$ they die out and the FM amplification is stable. $P_{\mathrm{th}}$ is inversely proportional to the quantum defect and the saturated amplifier gain. $P_{\mathrm{th}}$ is inversely proportional to the quantum defect $\left(\mathrm{q}_{\mathrm{D}}\right)$ and the saturated amplifier gain $\left(\mathrm{g}_{\mathrm{s}}\right)$. It is proportional to $\left(\lambda_{0} / \mathrm{D}_{0}\right)^{2}$, in good agreement with experimental data (as shown in Fig. 5) and Ref. [23].

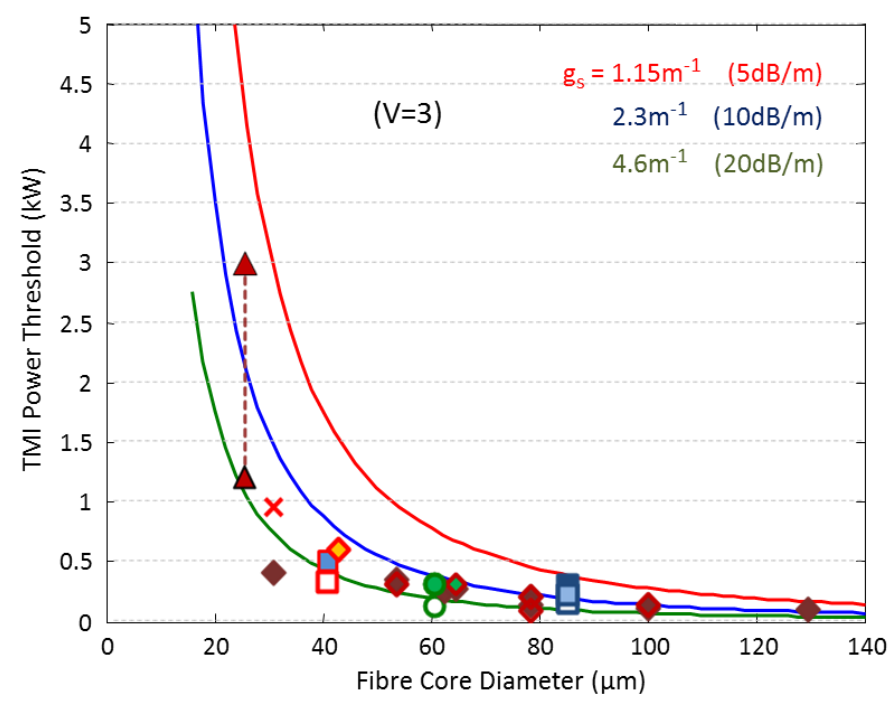

Fig. 5: TMI threshold $\left(P_{\text {thr }}\right)$ versus $D_{0}$ for different FM gain coefficients $\left(g_{\mathrm{s}}\right)$ (Fibre V=3) (included are experimental data from literature, see Ref. [1] and references therein. Red triangle data: from Ref. [6]).

Fig. 5 plots the TMI threshold as a function of fibre core diameter, for different gain coefficients. The step-index fibre Vnumber in all cases is assumed equal to 3 and the transverse perturbation corresponds to $L P_{11}$. The pump and signal wavelengths are $1060 \mathrm{~nm}$ and $976 \mathrm{~nm}$, respectively. It is shown that for the same output power, shorter amplifier lengths (larger gain coefficients) result in lower TMI thresholds compared to longer amplifier lengths (lower gain coefficient). In a fiber amplifier, local gain coefficient and heat load vary significantly along the length (see Fig. 3(c) \& (d)). Given that the main contribution to the total TMI gain comes from a highly localised region (see Fig. 4(c)), saturated gain $g_{s}$ in Eqn. (23) reflects the local gain coefficient, rather than its average value.

\section{SUMMARY}

Mode instabilities in fiber amplifiers are analyzed by a new approach, considering the stability of the steady state FM amplification in the presence of transverse amplitude and/or phase perturbations, taking into account the effects of 
population inversion and thermal loading due to quantum-defect heating. We observe that population inversion contribution to instability gain is dominant at low powers and high inversion. Under high powers and low inversion (high amplifier saturation) the thermal effects dominate the instability behavior. A simple and easy to interpret TMI power threshold formula is derived for the first time.

Acknowledgements: This project was supported by the Royal Academy of Engineering under the Research Chairs and Senior Research Fellowships Scheme.

\section{REFERENCES}

[1] M.N. Zervas and C.A. Codemard, "High Power Fiber Lasers: A Review," J. Select. Top. Quantum Electron., 20, 0904123 (2014).

[2] T. Eidam,, C. Wirth, C. Jauregui, F. Stutzki, F. Jansen, H.-J. Otto, O. Schmidt, T. Schreiber, J. Limpert, and A. Tünnermann, "Experimental observations of the threshold-like onset of mode instabilities in high power fiber amplifiers" Opt. Express, 1913218 (2011).

[3] M. Karow, H. Tünnermann, J. Neumann, D. Kracht, and P. Weßels, "Beam quality degradation of a single-frequency Yb-doped photonic crystal fiber amplifier with low mode instability threshold power," Opt. Lett. 37, 4242 (2012).

[4] R. Tao, P. Ma, X. Wang, P. Zhou, and Zejin Liu, " $1.3 \mathrm{~kW}$ monolithic linearly polarized single-mode master oscillator power amplifier and strategies for mitigating mode instabilities," Photon. Res. 3, 86 (2015).

[5] N. Haarlammert, B. Sattler, A. Liem, M. Strecker, J. Nold, T. Schreiber, R. Eberhardt, A. Tünnermann, K. Ludewigt, and M. Jung, "Optimizing mode instability in low-NA fibers by passive strategies," Opt. Lett. 40, 2317 (2015).

[6] F. Beier, C. Hupel, J. Nold, S. Kuhn, S. Hein, J. Ihring, B. Sattler, N. Haarlammert, T. Schreiber, R. Eberhardt, and A. Tünnermann, "Narrow linewidth, single mode $3 \mathrm{~kW}$ average power from a directly diode pumped ytterbiumdoped low NA fiber amplifier," Opt. Express 24, 6011 (2016).

[7] C. Jauregui, H-J Otto, F. Stutzki, J. Limpert1,2 and A. Tünnermann, "Simplified modelling the mode instability threshold of high power fiber amplifiers in the presence of photodarkening," Opt. Express 23, 20203 (2015).

[8] K. Brar, J. Henrie, S. Courtney, M. Savage-Leuchs, R. Afzal, E. Honea, "Threshold power and fiber-degradationinduced modal instabilities in high-power fiber amplifiers based on large-mode-area fibers," Proc. SPIE 8961, 89611R (2014).

[9] M. Kuznetsov, O. Vershinin, V. Tyrtyshnyy, and O. Antipov, "Low-threshold mode instability in Yb3+-doped fewmode fiber amplifiers," Opt. Express 22, 29714 (2014).

[10] K.-H. Lee, K. Lee, Y. Kim, Y.-H. Cha, G. Lim, H. Park, H. Cho, and D-Y. Jeong, "Transverse mode instability induced by stimulated Brillouin scattering in a pulsed single-frequency large-core fiber amplifier," Appl. Opt. 54, 189 (2015).

[11] C. Jauregui, T. Eidam, J. Limpert, and A. Tünnermann, "Impact of modal interference on the beam quality of highpower fiber amplifiers," Opt. Express 19, 3258 (2011).

[12] A.V. Smith and J. J. Smith, "Mode instability in high power fiber amplifiers," Opt. Express 19, 10180-10192 (2011).

[13] C. Jauregui, T. Eidam, H.-J. Otto, F. Stutzki, F. Jansen, J. Limpert, and A. Tunnermann, "Physical origin of mode instabilities in high-power fiber laser systems," Opt. Express 20, 12912-12925 (2012).

[14] A.V. Smith and J. J. Smith, "Steady-periodic method for modelling mode instability in fiber amplifiers," Opt. Express 21, 2606-2623 (2013).

[15] K.R. Hansen, T. T. Alkeskjold, J. Broeng, and J. Lægsgaard, "Theoretical analysis of mode instability in highpower fiber amplifiers," Opt. Express 21, 1944-1971 (2013).

[16] L. Dong, "Stimulated thermal Rayleigh scattering in optical fibers," Opt. Express 21, 2642-2656 (2013).

[17] B.G. Ward, C. Robin, and I. Dajani, "Origin of thermal modal instabilities in large mode area fiber amplifiers," Opt. Express 20, 11407-11422 (2012).

[18] S. Naderi, I. Dajani, T. Madden, and C. Robin, "Investigations of modal instabilities in fiber amplifiers through detailed numerical simulations," Opt. Exp. 21, 16111-16129 (2013).

[19] R. Tao, P. Ma, X. Wang, P. Zhou, and Z. Liu, "Study of Wavelength Dependence of Mode Instability Based on a Semi-Analytical Model,” J. Quantum Electron. 51, 1600106 (2015). 
[20] A. Fotiadi, O. Antipov, M. Kuznetsov, and P. Mégret, "Refractive index changes in rare earth-doped optical fibers and their applications in all-fiber coherent beam combining," in Coherent Laser Beam Combining, A. Brignon, ed. (John Wiley \& Sons, 2013), chap. 7, pp. $193-230$.

[21] M.N. Zervas, "TMI Threshold in High Power Fiber Amplifiers", paper SoW2H.2, OSA Advanced Photonics Congress (2016).

[22] A.W. Snyder and J.D. Love, Optical Waveguide Theory, Chapman and Hall, London, (1980- 1985).

[23] B.G. Ward, "Maximizing power output from continuous-wave single-frequency fiber amplifiers" Opt. Lett., 40, 542 (2015). 